\title{
Descriptors of intrinsic hydrodynamic thermal transport: screening a phonon database in a machine learning approach
}

\author{
Pol Torres, ${ }^{1,2,3}$ Stephen $\mathrm{Wu},{ }^{4}$ Shenghong Ju, ${ }^{5,1}$ Chang Liu, $, 6,4$ \\ Terumasa Tadano, ${ }^{7}$ Ryo Yoshida, ${ }^{4,6}$ and Junichiro Shiomi ${ }^{1}$ \\ ${ }^{1}$ Department of Mechanical Engineering, The University of Tokyo, 7-3-1 Hongo, Bunkyo, Tokyo, 113-8656, Japan \\ ${ }^{2}$ EURECAT, Technology Center of Catalonia, Applied Artificial Intelligence, 08290 Cerdanyola, Barcelona, Spain \\ ${ }^{3}$ Departament de Física, Universitat Autònoma de Barcelona (UAB), \\ Campus de Bellaterra, 08193 Bellaterra, Barcelona, Spain \\ ${ }^{4}$ Research Organization of Information and Systems, The Institute of Statistical Mathematics (ISM), \\ 10-3 Midori-cho, Tachikawa, Tokyo 190-8562, Japan \\ ${ }^{5}$ China-UK Low Carbon Collage, Shanghai Jiao Tong University, Shanghai 201306, China \\ ${ }^{6}$ Center for Materials Research by Information Integration (CMI2), \\ Research and Services Division of Materials Data and Integrated System (MaDIS), \\ National Institute for Materials Science (NIMS), 1-2-1 Sengen, Tsukuba, Ibaraki 305-0047, Japan \\ ${ }^{7}$ Research Center for Magnetic and Spintronic Materials, \\ National Institute for Materials and Science, Tsukuba, Japan
}

(Dated: October 26, 2021)

\begin{abstract}
Machine learning techniques are used to explore the intrinsic origins of the hydrodynamic thermal transport and to find new materials interesting for science and engineering. The hydrodynamic thermal transport is governed intrinsically by the hydrodynamic scale and the thermal conductivity. The correlations between these intrinsic properties and harmonic and anharmonic properties, and a large number of compositional (290) and structural (1224) descriptors of 131 crystal compound materials are obtained, revealing some of the key descriptors that determines the magnitude of the intrinsic hydrodynamic effects, most of them related with the phonon relaxation times. Then, a trained black-box model is applied to screen more than 5000 materials. The results identify materials with potential technological applications. Understanding the properties correlated to hydrodynamic thermal transport can help to find new thermoelectric materials and on the design of new materials to ease the heat dissipation in electronic devices.

PACS numbers:
\end{abstract}

\section{INTRODUCTION}

From the Boltzmann transport equation (BTE) for phonons it is well known that the thermal conductivity of a crystalline material is mainly determined by the phonon scattering. The stronger the scattering is, the higher the thermal resistance is. Phonons can scatter with each other anharmonically, against boundaries, with impurities and defects. The BTE states that when the phonon distribution is perturbed from its equilibrium, it relaxes to the equilibrium through the scattering events [1]

When studying the thermal transport in bulk materials and under slow heating conditions, the Fourier's law provides a simple expression for the diffusive thermal transport:

$$
\mathbf{Q}=-\kappa \nabla T
$$

where $\mathbf{Q}$ is the heat flux, $\kappa$ the thermal conductivity and $T$ the temperature. In recent experiments at the micro/nano scale and/or using fast and/or large thermal gradient, it has been observed that not all thermal transport can be explained by the Fourier's law [5 11]. In such situations, other transport phenomena such as ballistic transport and Lévy flights [12, 13] or hydrodynamic transport appear $14-16]$. In this article we will focus on the latter transport regime.

In the second half of the last century, Guyer and Krumhansl introduced an expression of the thermal transport beyond the Fourier's law to describe the hydrodynamic thermal transport [1, 17]:

$$
\tau \dot{\mathbf{Q}}+\mathbf{Q}=-\kappa \nabla T+\ell^{2} \nabla^{2} \mathbf{Q}
$$

where $\tau$ is the phonon mean free time and $\ell$ is the hydrodynamic scale. Both of these are the values averaged over the entire phonon spectrum [4, 16]. Eq. (2) is a general hydrodynamic heat flux equation that includes memory (time derivative term) and non-local effects (Laplacian term), and it has been used successfully to describe recent experiments [16, 18] that could not be reproduced only by Fourier's law. 
It is known that the thermal hydrodynamic effects are related with the relative importance of Normal (N) scattering (where phonon-phonon collisions conserve momentum) in front of restive (R) scattering (where phononphonon collisions do not conserve momentum, like Uumklapp scattering or mass deffect), but little is known about the intrinsic origin of these phenomena. In addition, as described by Eq. (2), the temporal scale and the geometry will also contribute to this effect. To elucidate the key structural/chemical factors that determine the hydrodynamic thermal transport, leaving aside the temporal and geometrical scales, in this paper we employ machine learning techniques. A Python open source platform of materials informatics that we have developed called XenonPy [19] is used to generate compositional and sctructural descriptors. By providing only the Materials Project ID of a certain material [20, 21], the tool provides 290 compositional descriptors and 1224 structural descriptors like the polarizability, lattice constant, and Van Der Waals radius, etc.

\section{METHODOLOGY}

In this work we perform full first-principles calculations for 131 materials. The interatomic force constants from 94 of those materials are provided by A. Togo [22], 18 are from the AlmaBTE database 23] and 20 are calculated by ourselves. Our DFT calculations are performed using the Vienna Ab initio Simulation Package (VASP) in the framework of the projector augmented wave (PAW) potentials in the Perdew-Burke-Ernzerhof (PBE) approximation. The energy cutoff was fixed at a value $10 \%$ higher than the value specified in the pseudopotential file. The atom displacements to calculate the harmonic and anharmonic interatomic force constants are provided by the Phonopy 24] and Phono3py [25] codes respectively.

The parameters that describe the hydrodynamic thermal transport from Eq. (2), mainly $\kappa, \tau$ and $\ell$ are computed from the first-principles calculations beyond the relaxation-time approximation (RTA) by using Phono3py and the KCM code 26]. All the calculations are performed at $300 \mathrm{~K}$. From the calculations, we have obtained extra first-principles descriptors like the averaged Normal and resistive (Umklapp plus mass variance/impurity) relaxation times $\left(\tau_{N}, \tau_{R}\right)$, specific heat $C$, and the group velocity $v$. In recent articles, it has been described that other parameters such as the maximum frequency of the phonon dispersion relations $\left(\omega_{\max }\right)$, the Grüneisen parameter, the volume of the unit cell $(V)$, and the scattering phase space $(S P S)$ show a good correlation with the thermal conductivity [27, 28]. These are harmonic and quasi-harmonic properties but not all of them are equally easy to calculate. While the maximum frequency is obtained with a single harmonic calculation, evaluation of the Grüneisen parameter requires a few calculations with different volumes. The $S P S$ is defined as:

$P_{3}^{ \pm}(\mathbf{q} j)=\frac{1}{N_{\mathbf{q}}} \sum_{q 1, q 2, j 1, j 2} \delta\left(\omega_{q, j} \pm \omega_{q 1, j 1}-\omega_{q 2, j 2}\right) \delta_{q \pm q 1, q 2+G}$

where $\omega$ and $\mathbf{q}$ are the phonon frequency and the crystalline momentum of each phonon involved in the threephonon interaction. Therefore, although $S P S$ is a harmonic property, it requires exploration of all the threephonon scattering processes, which takes a some time.

The recent works have found good correlation between $w_{\max }$ and $\kappa[27,28]$, and from our experience we know that in several cases $\kappa$ shows a good correlation with $\ell$. The correlation of $w_{\max }$ and $\kappa$ can be broken in materials with high frequency optical bands. Those bands are usually flat and therefore the velocity is small and does no contribute significantly to the thermal conductivity. For instance, lithium hydride $(\mathrm{LiH})$ and lithium fluoride $(\mathrm{LiF})$ have $\omega_{\max } \sim 32 \mathrm{THz}(\sim 20 \mathrm{THz})$ and $\kappa=22 \mathrm{~W} / \mathrm{mK}(14 \mathrm{~W} / \mathrm{mK})$ respectively [29] while silicon $(\mathrm{Si})$ has $\omega_{\max } \sim 16 \mathrm{THz}$ and $\kappa=145 \mathrm{~W} / \mathrm{mK}$ at $300 \mathrm{~K}[30$ ].

In the effort of finding a new descriptor including the velocity to avoid the effect of high frequency optical bands, we propose two candidates. The first one is equivalent to the Landauer thermal conductance expressed in the $\mathbf{q}$ space considering the transmission $T=1$ for all the modes:

$$
G_{\text {max }}=\frac{1}{N_{\mathbf{q}} V} \sum_{\mathbf{q}, j} \frac{\hbar \omega}{2 \pi k_{B} T^{2}} \frac{e^{\hbar \omega / k_{B} T}}{\left(e^{\hbar \omega / k_{B} T}-1\right)^{2}} \mathbf{v},
$$

where $N$ and $V$ are number of q-points in the mesh sampling and the volume of the unit cell, respectively. The second descriptor is a pseudo thermal conductivity defined in the following. We know that the thermal conductivity under the RTA can be expressed as:

$$
\kappa^{\alpha \beta}=\frac{1}{N_{\mathbf{q}} V} \sum_{\mathbf{q}, j} C_{\mathbf{q}, j} v_{\mathbf{q}, j}^{\alpha} v_{\mathbf{q}, j}^{\beta} \tau_{\mathbf{q}, j},
$$

where $\alpha, \beta$ are cartesian cordinates, $C_{\mathbf{q}, j}$ the specific heat and $\tau_{\mathbf{q}, j}$ the relaxation time. The latter is calculated from the phonon-phonon scattering rate as $\tau_{\mathbf{q}, j}=\hbar / 2 \Gamma$, where

$$
\begin{array}{r}
\Gamma_{\mathbf{q} j}=\frac{\pi \hbar}{2 N_{\mathbf{q} j}} \sum_{\mathbf{q}^{\prime} j^{\prime} \mathbf{q}^{\prime \prime} j^{\prime \prime}} \frac{\left|\Phi\left(\mathbf{q} j \mathbf{q}^{\prime} j^{\prime} \mathbf{q}^{\prime \prime} j^{\prime \prime}\right)\right|^{2}}{8 \omega_{\mathbf{q} j} \omega_{\mathbf{q}^{\prime} j^{\prime}} \omega_{\mathbf{q}^{\prime \prime} j^{\prime \prime}}} . \\
{\left[\left(1+n_{\mathbf{q}^{\prime} j^{\prime}}+n_{\mathbf{q}^{\prime \prime} j^{\prime \prime}}\right) \delta\left(\omega_{\mathbf{q} j}-\omega_{\mathbf{q}^{\prime} j^{\prime}}-\omega_{\mathbf{q}^{\prime \prime} j^{\prime \prime}}\right)\right.} \\
\left.+2\left(n_{\mathbf{q}^{\prime} j^{\prime}}-n_{\mathbf{q}^{\prime \prime} j^{\prime \prime}}\right) \delta\left(\omega_{\mathbf{q} j}+\omega_{\mathbf{q}^{\prime} j^{\prime}}-\omega_{\mathbf{q}^{\prime \prime} j^{\prime \prime}}\right)\right]
\end{array}
$$


and $\left|\Phi\left(\mathbf{q q}^{\prime} \mathbf{q}^{\prime \prime}\right)\right|^{2}$ is the three-phonon scattering matrix. If we approximate $\left|\Phi\left(\mathbf{q q}^{\prime} \mathbf{q}^{\prime \prime}\right)\right|^{2} / 8 \omega_{\mathbf{q} j} \omega_{\mathbf{q}^{\prime} j^{\prime}} \omega_{\mathbf{q}^{\prime \prime} j^{\prime \prime}} \rightarrow 1 / m^{2}$, then

$$
\Gamma_{\mathbf{q}}=\frac{\pi \hbar}{2 m^{2}}\left(W_{\mathbf{q}, j}^{-}+2 W_{\mathbf{q}, j}^{+}\right) .
$$

The magnitude $W_{\mathbf{q}, j}^{ \pm}$is the temperature dependent $S P S$ (TSPS), which as well as the $S P S$, can be obtanied directly from Phono3py [25]. With these definitions, we introduce the pseudo thermal conductivity as:

$\kappa_{\text {pseudo }}^{\alpha \beta}=\frac{1}{N_{\mathbf{q}} V} \sum_{\mathbf{q}, j} C_{\mathbf{q}, j} v_{\mathbf{q}, j}^{\alpha} v_{\mathbf{q}, j}^{\beta}\left[\frac{\pi}{m^{2}}\left(W_{\mathbf{q}, j}^{-}+2 W_{\mathbf{q}, j}^{+}\right)\right]^{-1}$.

\section{RESULTS AND DISCUSSION}

The values of $\ell, \kappa$ and all the descriptors described in the previous section has been calculated for the 131 materials from first-principles and with XenonPy.

The first-principles calculations show that the materials with stronger intrinsic hydrodynamic behaviour (larger $\ell$ ) are those made of carbon, such as diamond (mp-66), lonsdaleite (mp-47), and other carbon allotropes (mp-611426 and mp-616440), as well as BAs in wurtzite(WZ) and zinc-blend(ZB) structures. The former carbon based materials have a thermal conductivity of around $2500 \mathrm{~W} / \mathrm{mK}$ while the hydrodynamic scale ranges from $800-1000 \mathrm{~nm}$. In comparison with BAs, the $\kappa$ is smaller while $\ell$ is larger. More explicity, WZ(ZB) BAs have a thermal conductivity of $\sim 1250 \mathrm{~W} / \mathrm{mK}(\sim 1500 \mathrm{~W} / \mathrm{mK})$ and $\ell \sim 1100 \mathrm{~nm}(\sim 1400 \mathrm{~nm})$. Another case is BN, which has a large thermal conductivity $(1200 \mathrm{~W} / \mathrm{mK})$ but smaller $\ell$ $(319 \mathrm{~nm})$ than BAs in wurtzite (WZ) and zinc-blend (ZB) phases. Those materials with large $\ell$ are interesting from the viewpoint of studying the experimental signatures of the hydrodynamic thermal transport, as they also exhibit a high relative importance of $\mathrm{N}$ versus resistive scattering processes, as reported in several works [31, 32]. Also, these materials can be usefull to study the improvement of heat release in hot spots in electronic devices due to fast operation rates, as hydrodynamic effects near to the edges of electronic circuits create a vorticity that helps to increase the heat dissipation due to an effective change of the thermal conductivity [16].

The list of 131 materials used in this work corresponds to some of the most studied and useful insulators and seminconductors for thermal management and thermoelectrics 33 38]. From the first-principles calculations it can be observed that most of them fulfil the condition

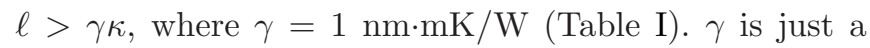

TABLE I: Materials with $\ell>\gamma \kappa . \kappa$ is expressed in $\mathrm{W} / \mathrm{mK}$ and $\ell$ in $\mathrm{nm}$. More materials fulfilling this condition can be found in the APPENDIX.

\begin{tabular}{|c|c|c|c|c|}
\hline Material & $\kappa$ & $\ell$ & Material & $\kappa$ \\
\hline $\mathrm{AlP}(\mathrm{mp}-1550)$ & 101.5 & 151.7 & $\mathrm{GaN}(\mathrm{mp}-830)$ & 201.4202 .3 \\
\hline $\mathrm{BeS}(\mathrm{mp}-422)$ & $\overline{164.4}$ & 173.2 & $\mathrm{BeTe}(\mathrm{mp}-252)$ & $\begin{array}{ll}200 & 351.7\end{array}$ \\
\hline $\begin{array}{l}\mathrm{Bi}_{2} \mathrm{Te}_{3} \\
(\mathrm{mp}-1227339)\end{array}$ & 1.5 & 10 & $\mathrm{NaBr}(\mathrm{mp}-22916)$ & 11.6 \\
\hline $\mathrm{KBr}(\mathrm{mp}-23251)$ & 3.3 & 17.1 & $\mathrm{BaSe}(\mathrm{mp}-1253)$ & $\begin{array}{ll}11.5 & 53.2\end{array}$ \\
\hline $\mathrm{BaTe}(\mathrm{mp}-1000)$ & 13.4 & 63.8 & AgI(mp-22925) & 42.6 \\
\hline $\mathrm{CdS}(\mathrm{mp}-2469)$ & 25.6 & 111.3 & $\mathrm{CdSe}(\mathrm{mp}-2691)$ & 59.4 \\
\hline $\mathrm{CdTe}(\mathrm{mp}-406)$ & 6 & 42.6 & $\mathrm{CuBr}(\mathrm{mp}-22913)$ & 11.2 \\
\hline GaAs(mp-2534) & 36.5 & 94.3 & $\mathrm{GaP}(\mathrm{mp}-2490)$ & $84.7 \quad 180.0$ \\
\hline GaSb(mp-1156) & 40.8 & 145.4 & $\begin{array}{c}\text { InAs } \\
(\mathrm{mp}-20305)\end{array}$ & $\begin{array}{ll}24.6 & 97.0\end{array}$ \\
\hline $\operatorname{InP}(\mathrm{mp}-20351)$ & 77.0 & 426.2 & $\begin{array}{c}\operatorname{InSb} \\
(\mathrm{mp}-20012)\end{array}$ & 15.6 \\
\hline $\mathrm{MgTe}(\mathrm{mp}-13033)$ & 15.8 & 88.8 & $\begin{array}{c}\mathrm{ZnS} \\
(\mathrm{mp}-10695)\end{array}$ & $23.4 \quad 75.6$ \\
\hline$\overline{Z n S e}(\mathrm{mp}-1190)$ & 17.5 & 52.4 & $\mathrm{ZnTe}(\mathrm{mp}-2176)$ & $15.9 \quad 96.2$ \\
\hline $\mathrm{AgI}(\mathrm{mp}-22894)$ & 1 & 18.6 & $\operatorname{AlAs}(m p-8881)$ & 82.3198 .6 \\
\hline $\mathrm{AlP}(\mathrm{mp}-8880)$ & 83.1 & 126.5 & $\begin{array}{c}\text { AlSb } \\
(\mathrm{mp}-1018100)\end{array}$ & 84.7 \\
\hline $\mathrm{CdS}(\mathrm{mp}-672)$ & 19.4 & 82.1 & $\mathrm{CdSe}(\mathrm{mp}-1070)$ & 42.8 \\
\hline $\mathrm{CdTe}(\mathrm{mp}-12779)$ & 4.6 & 42.4 & $\mathrm{CuI}(\mathrm{mp}-569346)$ & 46.1 \\
\hline $\mathrm{GaP}(\mathrm{mp}-8882)$ & 79.2 & 190.3 & $\begin{array}{c}\text { GaSb } \\
(\mathrm{mp}-1018059)\end{array}$ & 26.8 \\
\hline $\operatorname{InAs}(\mathrm{mp}-1007652)$ & 18.7 & 93.6 & $\begin{array}{c}\text { InP } \\
(\mathrm{mp}-966800)\end{array}$ & 67.6 \\
\hline $\operatorname{InSb}(\mathrm{mp}-1007661)$ & 10.8 & 72 & MgTe(mp-1039) & $\begin{array}{ll}11.8 & 75.3 \\
\end{array}$ \\
\hline $\mathrm{ZnTe}(\mathrm{mp}-8884)$ & 13.1 & 82.6 & $\mathrm{Sn}(\mathrm{mp}-117)$ & $\begin{array}{ll}28.3 & 108.8\end{array}$ \\
\hline $\begin{array}{l}\text { TlMgLa } \\
\text { (mp-962063) }\end{array}$ & 9.3 & 54.2 & $\operatorname{InN}(\mathrm{mp}-22205)$ & 110.1226 .3 \\
\hline $\begin{array}{l}\text { AlSb } \\
(\mathrm{mp}-2624)\end{array}$ & 131.5 & 417.3 & $\begin{array}{c}\text { InN } \\
(\mathrm{mp}-20411)\end{array}$ & 108.7235 .4 \\
\hline $\operatorname{AlAs}(\mathrm{mp}-2172)$ & 114.7 & 237 & & \\
\hline $\mathrm{Be}_{2} \mathrm{C}(\mathrm{mp}-1569)$ & 149.6 & 213 & $\begin{array}{c}\mathrm{B}_{2} \mathrm{AsP} \\
(\mathrm{mp}-1008528)\end{array}$ & $\overline{433}$ \\
\hline
\end{tabular}

factor to evaluate the difference in the order of magnitude and units between $\kappa$ and $\ell$. Taking into account this observation, from the data of the studied 131 materials, we use machine learning models to look for materials with some interesting hydrodynamic properties, such as materials with $\ell$ values higher than $1000 \mathrm{~nm}$ (similar to C-based materials or BAs) or materials fulfilling the condition $\ell>\gamma \kappa$.

Recently various machine learning techniques have been used to predict the thermal conductivity, like Bayesian optimization 22], Gaussian process regression [27] or transfer learning [28]. In this work we have examined different machine learning models trained us- 
ing the scikit-learn library in Python. Three types of ensemble learning with regression tree models and neural networks with different combination of layers were considered. The corresponding packages are ExtraTreesRegressor [39], RandomForestRegressor [40], and GradientBoostingRegressor [41] and XenonPy. Hyperparameters for each type of model were selected using 5 -fold crossvalidation based on the GridSearchCV package, using in all cases $25 \%$ of data for test. We selected negative mean squared error as the scoring metric. Finally, the model with the lowest root mean squared error (RMSE) on the test data was selected for the high-throughput screening of $\ell$ and $\kappa$.
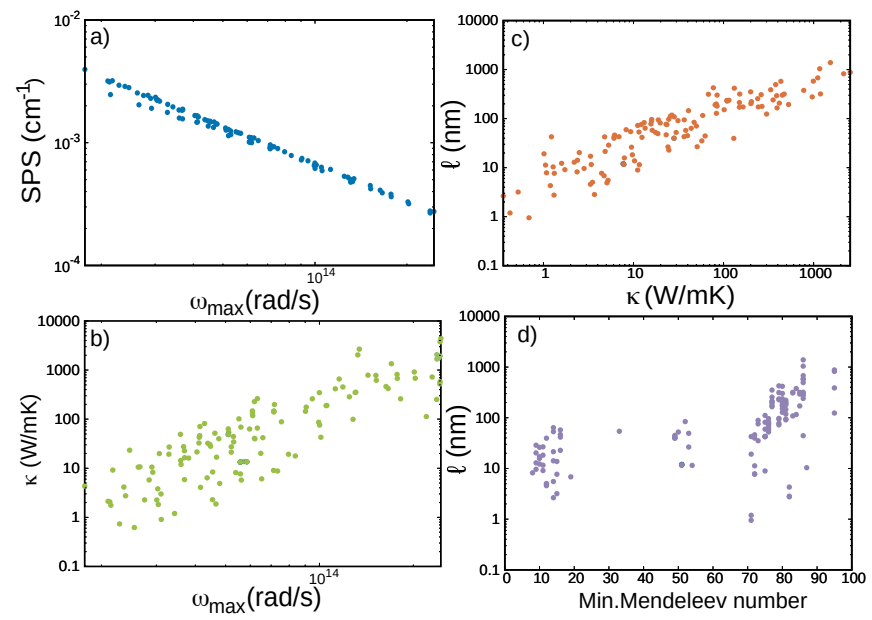

FIG. 1: Scatter plot of relevant correlations found in the present work for the 131 materials set. a) $S P S$ as function of $\left.\omega_{\max } . \mathbf{b}\right) \kappa$ as function of $\left.\omega_{\max } . \mathbf{c}\right) \ell$ as function of $\kappa$. d) $\ell$ as function of Min. Mendeleev number. For $\ell$ and $\kappa$ the median value of the three components have been considered.

Considering all the descriptors described in the previous section, those calculated from first-principles and those obtained directly from XenonPy, we have calculated the Pearson's correlation matrix and obtained the corresponding absolute correlation coefficients. By examining the correlation matrix for all the 131 materials we can observe a few interesting things. First of all, we see that the $S P S$ have a correlation of 0.996 with $w_{\max }$ (Fig. 1 1a). From the harmonic point of view, we have also confirmed that $w_{\max }$ strongly correlates with $\kappa$ (Fig. 1 b), with a correlation coefficient of 0.86 , while $v$ and $G_{\max }$ with values of 0.78 and 0.71 respectively are better than $V(0.53)$. Those correlations for the hydrodynamic scale $\ell$ are worse. In the case of $w_{\max }$ is 0.59 , while for $v$ and $G_{\max }$ are 0.48 and 0.33 respectively. Unfortunately the correlation coefficient between $\ell$ and the pseudo thermal conductivity is lower than 0.5
(0.48). On the other hand, the correlation between $\kappa$ and $\ell$ is quite high (0.88) (Fig. 1T), as both parameters have a strong correlation with the phonon relaxation times according to their definitions [4, 16]. We also observe that $\ell$ strongly correlates with the total relaxation time $\tau(0.92)$ and the Normal relaxation time $\tau_{N}(0.90)$, as by definition $\ell=\ell\left(\tau, \tau_{N}\right)$ [4]. For $\kappa$ these correlations are weaker, 0.72 and 0.73 respectively. In addition to the Pearson's correlation coefficients, which accounts for linear correlations, the Maximal Information Coefficient (MIC) has been also calculated, which also consider non-linear correlations.

TABLE II: Top descriptors with MIC correlation with $\ell$ higher than 0.7 .

\begin{tabular}{lc}
\hline Descriptor & Correlation with $\ell$ \\
\hline Min. Mendeleev number & 0.79 \\
\hline Ave. dipole polarizability & 0.76 \\
\hline Max. dipole polarizability & 0.76 \\
\hline Max. polarizability & 0.72 \\
\hline Min. Allen energy & 0.71 \\
\hline Var. Mendeleev number & 0.7 \\
\hline Min. atomic number & 0.7 \\
\hline Min. atomic weight & 0.7 \\
\hline Max. Van Der Waals radius (Alvarez) & 0.7 \\
\hline
\end{tabular}

The descriptors with a MIC with $\ell$ higher than 0.7 are summarized in Table II Regarding those descriptors we can observe that none of the harmonic or quasi-harmonic descriptors are in the list, and that the highest correlation with $\ell$ is found for the minimum Mendeleev number (0.79) (Fig. 1 $1 \mathrm{~d}$ ). While the meaning of this compositional descriptors is self-explained by the names, the Min, Max corresponds to Min/Max values of the descriptor for each unique atom of the compound, and the Ave to the average of the descriptor of all the atoms of the compound. This information provides valuable physical insight that is supported by previous works. As detailed before, the hydrodynamic thermal transport is strongly related to the phonon relaxation times and to several compositional descriptors. Several works have determined analytical expressions for the phonon relaxation times [30, 42 47], showing that those magnitudes are directly related with the material density $\rho$, group velocity, and frequency for the case of Normal and Umklapp scattering, in agreement with some of the higher correlated descriptors for $\kappa$. In the case of impurity/mass defect scattering the analytic expresions show the relation to the atomic masses of the atoms of a compound [48, 49]. This also agrees with the high correlation of $\kappa$ and $\ell$ with the atomic number and atomic weight. Our calculations have also identified the 
polarizability as a key factor to determine the intrinsic hydrodynamic effects. A recent work have shown that by strain engineering it is possible to tune the polarizability of a material and modify the thermal conductivity [50], revealing the high correlation between both magnitudes. In our case the correlation of the Max. polarizability with $\kappa$ is 0.63 , while for $\ell$ is 0.76 . Therefore it is expected that modifications in the polarizability will have a big influence on $\ell$.

The descriptors for $\kappa$ showing MIC higher than 0.7 can be found in Table III] Note that here we have omitted the $S P S$, showing also a correlation of 0.7 , as for our calculated materials it is almost equivalent to $\omega_{\max }$ but it is much more expensive computationally (Fig. 1 a a). In materials with large band gaps this might not be true.

TABLE III: Top descriptors with MIC correlation with $\kappa$ higher than 0.7 .

\begin{tabular}{lc}
\hline Descriptor & Correlation with $\kappa$ \\
\hline$w_{\max }$ & 0.86 \\
\hline Min. atomic weight & 0.79 \\
\hline Min. atomic number & 0.79 \\
\hline$v$ & 0.78 \\
\hline Ave. heat of formation & 0.77 \\
\hline Ave. GS energy & 0.71 \\
\hline$G_{\max }$ & 0.71 \\
\hline
\end{tabular}

By using the descriptors from Table $\amalg$ and Table $\amalg$ we first create machine learning models for $\ell$ and $\kappa$ with our known 131 materials calculated from first-principles.

A neural network with 6 layers (with 9-69-59-30-13-1 neurons in each layer) has been selected for $\ell$, and a neural network with 5 layers (with 7-59-52-17-1 neurons in each layer) for $\kappa$ as the models with lower RMSE. The best models showed an RMSE value of $2.64 \mathrm{~nm}$ for $\ell$ and $5.47 \mathrm{~W} / \mathrm{mK}$ for $\kappa$ respectively. In Fig. 2 the firstprinciples values of $\kappa$ (top) and $\ell$ (bottom) are represented versus the prediction obtained from informatics techniques. It can be observed that the predictions are slightly better for high $\kappa$ values than for the lower ones. Low $\kappa$ materials are highly anharmonic and the present formalism might not be accurate enough. The straight line with slope of 1 represents an exact prediction. The calculated values of $\ell$ and $\kappa$ are averaged over the three axial components.

Now, we proceed to screen all the materials available in the phonon database of the Kyoto University [20, 21, 51, 52]. In the phonon database we can find the harmonic information for 10035 materials. From the initial list, we have removed those showing negative phonon bands, reducing the number to 5059. This harmonic information allows us to compute harmonic and quasi-harmonic properties such as $\omega_{\max }, G_{\max }, C, v$, $S P S$ and $\kappa_{\text {pseudo }}^{\alpha, \beta}$, in addition to the material density $\rho$ and volume $V$, for all the 5059 materials.
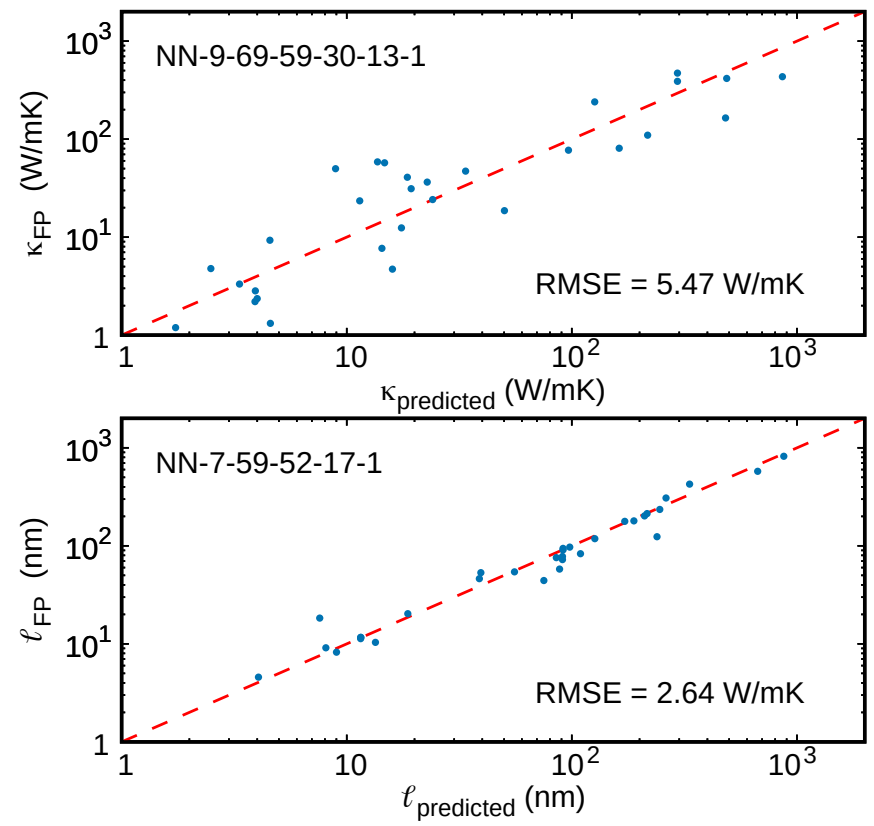

FIG. 2: Values of $\kappa$ and $\ell$ calculated by first-principles (FP) versus the prediction obtained by machine learning algorithms for the $25 \%$ test set. The straight line represents and ideal exact prediction.

TABLE IV: Validation set of materials with $\ell>\gamma \kappa$. $\kappa$ is expressed in $\mathrm{W} / \mathrm{mK}$ and $\ell$ in $\mathrm{nm}$.

\begin{tabular}{lcccccc}
\hline Material & $\kappa_{\mathbf{x}}$ & $\kappa_{\mathbf{y}}$ & $\kappa_{\mathbf{z}}$ & $\ell_{\mathbf{x}}$ & $\ell_{\mathbf{y}}$ & $\ell_{\mathbf{z}}$ \\
\hline $\mathrm{GeAs}_{2}(\mathrm{mp}-17524)$ & 8 & 2.6 & 2.4 & 56 & 102.9 & 82.9 \\
\hline $\mathrm{SiP}(\mathrm{mp}-2798)$ & 41.9 & 19.4 & 12.9 & 93.5 & 41 & 133.9 \\
\hline $\mathrm{BeP}_{2}(\mathrm{mp}-27148)$ & 73.5 & 71.5 & 57.5 & 92.9 & 91.4 & 105 \\
\hline $\mathrm{GeO}_{2}(\mathrm{mp}-470)$ & 63.6 & 63.6 & 101 & 93.8 & 93.8 & 95.5 \\
\hline
\end{tabular}

After screening over the 5059 materials, the models have found 147 compounds fulfilling the $\ell>\gamma \kappa$ criteria. From the whole list we have selected 5 materials to check the validity of the predictions from first-principles: $\mathrm{GeAs}_{2}, \mathrm{SiP}, \mathrm{BeP}_{2}$ and $\mathrm{GeO}_{2}$. The values of thermal conductivity and hydrodynamic scale calculated from firstprinciples for each axial component can be found in Table IV Due to the limited resources, all the 147 materials can not be calculated from first-principles. The full list of materials fulfilling the condition $\ell>\gamma \kappa$ from the phonon database according to our calculations can be found in the APPENDIX. 
From TableIV it can be observed that all the materials fullfil the condition $\ell>\gamma \kappa$ also from first-principles calculations. It is expected that, according to this condition, the materials could be useful for thermal management and/or thermoelectrics. In several works it has been analysed that the dominance of Normal phonon-phonon and electron-phonon scattering in front of Umklapp processes produces a fluid-like behaviour (hydrodynamics) that generates phonon drag and increases the Seebeck coefficient, and consequently an enhancement of the thermoelectric efficiency (ZT) [53 59]. In the case of SiP, E. Jiang et. al 60] showed that in its monolayer form can achieve a $\mathrm{ZT} \sim 0.8$ and a Seebeck coefficient of $3 \mathrm{mV} / \mathrm{K}$, opening the door to further improvements for nanoscale P-based materials. In the case of $\mathrm{BeP}_{2}$, its bulk $\mathrm{ZT}$ is around 1 , and in addition several works have highlighted the greatly tunable electronic properties that exhibit in its monolayer and penta-monolayer form 61, 62. GeAs 2 has been reported to be a material with ultralow thermal conductivity and high thermoelectric efficiency [63], showing a Seebeck coefficent higher than $0.3 \mathrm{mV} / \mathrm{K}$ with high carrier concentration. Finally, $\mathrm{GeO}_{2}$ has been examined in the last decades as material used to improve the thermoelectric efficiency of other materials by alloying with $\mathrm{Cu}, \mathrm{SiO}_{2}$ and $\mathrm{CdO}$ to create thin films 64 66. or coating conducting polymers with $\mathrm{GeO}_{2}$ nanoparticles [67]. Unfortunately, the models have not found any new material with high $\ell$ value above $1000 \mathrm{~nm}$.

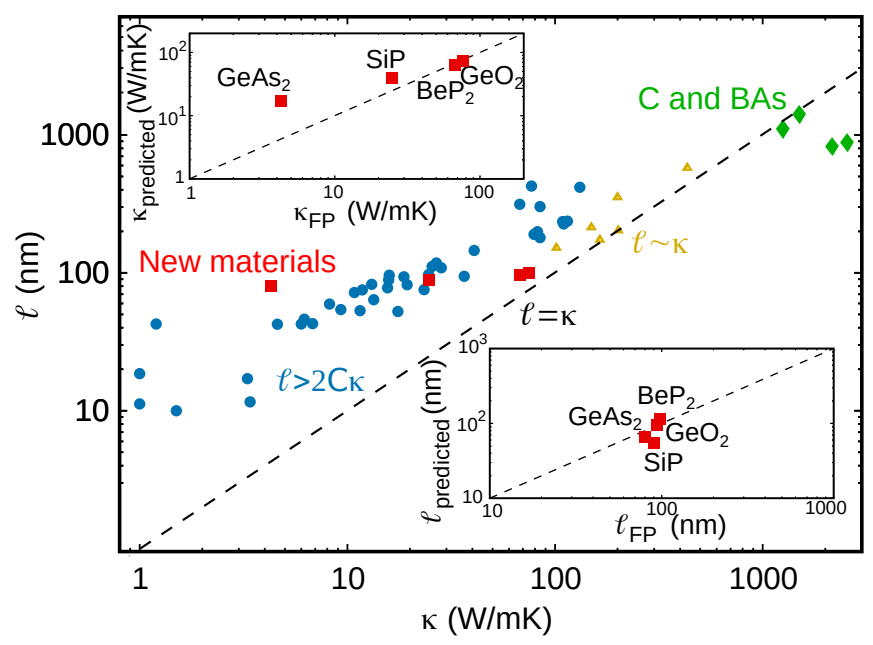

FIG. 3: Values of $\kappa$ and $\ell$ for the materials corresponding to Table I (blue circles), Table IV (red squares) and C and BN allotropes (green diamonds). The insets show the predicted $V S$ the first-principles (FP) calculations for $\kappa$ (top) and $\ell$ (bottom).

The results from Table \and Table IV are represented in Fig. 3] which seems to indicate that thermoelectric materials tend to exhibit a $\ell>\gamma \kappa$ behaviour. The insets show, for the materials of Table IV] the predicted values by the machine learning models as function of their values calculated from first-principles. The straight line represents the prefect prediction. It can be observed that for low values of $\kappa$ the prediction is worse than for high values. This might be because the present formalism used to calculate the thermal conductivity is not suitable to capture the high anharmonicity of low $\kappa$ materials, affecting the values used to train the models and therefore the predictions. In addition, materials with $\ell>1000 \mathrm{~nm}$ and below the $\ell=\gamma \kappa$ line would be the best candidates to improve heat dissipation in thermal management of electronics.

\section{CONCLUSIONS}

In conclusion, we have used XenonPy's structural and compositional material descriptors together with firstprinciples calculations to explore the hydrodynamic thermal transport. By using machine learning calculations, we have found that the structural descriptors are not relevant to study the hydrodynamic thermal transport, while there are 9 compositional descriptors with a correlation higher than 0.7 with $\ell$, the key parameter expressing the scale of phonon hydrodynamics. The calculations have also revealed that diamond and BAs and their allotropes have both high $\ell$. Those materials are the best candidates to identify hydrodynamic signatures from an experimental point of view. The second group of materials that have been considered are those having $\ell>\gamma \kappa$, which seems to be a good indicator to explore new materials to be used for thermal management and thermoelectricity. Here several materials have been found in the original data set, while the machine learning techniques identified 147 materials. From the identified samples, $\mathrm{BeP}_{2}, \mathrm{SiP}, \mathrm{GeAs}_{2}$ and $\mathrm{GeO}_{2}$ have been calculated from first-principles and used to validate the models, which according to recent works seem to have high thermoelectric performance, in agreement with our original guess. Although these materials have been already reported for this propose, the model found them without knowing of their existence a priori. Finally, the full list of values of $\kappa$ and $\ell$ of the materials from the original dataset and the 147 found materials with $\ell>\gamma \kappa$ detailed in the APPENDIX can be useful to look for new compounds for thermal management and thermoelectrics. 


\section{ACKNOWLEDGMENTS}

We acknowledge financial support of The Canon Foundation. This work was partially supported by JSPS KAKENHI (19H00744, 19H01132, 19H50820, 18K18017, 19K14902) and JST CREST (JPMJCR20Q3, JPMJCR19I2, JPMJCR19I1).

\section{APPENDIX}

In the next table all the values of thermal conductivity $\kappa$ and non-local length $\ell$ for the materials used for the training are displayed. The values that have been already showed in the main text are not included.

TABLE V: Values of $\kappa$ and $\ell$ of the full data set used for training the models. The averaged values of the three components are considered.

\begin{tabular}{ccc}
\hline Material & $\kappa(\mathrm{W} / \mathrm{mK})$ & $\ell(\mathrm{nm})$ \\
\hline $\mathrm{Ge}(\mathrm{mp}-32)$ & 58.6 & 116.0 \\
\hline $\mathrm{C}(\mathrm{mp}-66)$ & 2552.3 & 880.2 \\
\hline $\mathrm{LiCl}(\mathrm{mp}-22905)$ & 5.1 & 4.9 \\
\hline $\mathrm{LiBr}(\mathrm{mp}-23259)$ & 3.5 & 5.0 \\
\hline $\mathrm{LiI}(\mathrm{mp}-22899)$ & 3.3 & 4.6 \\
\hline $\mathrm{NaF}(\mathrm{mp}-682)$ & 50.9 & 26.7 \\
\hline $\mathrm{NaCl}(\mathrm{mp}-22862)$ & 9.7 & 18.3 \\
\hline $\mathrm{NaI}(\mathrm{mp}-23268)$ & 1.7 & 9.1 \\
\hline $\mathrm{KF}(\mathrm{mp}-463)$ & 7.9 & 15.8 \\
\hline $\mathrm{KCl}(\mathrm{mp}-23193)$ & 9.5 & 26.1 \\
\hline $\mathrm{KI}(\mathrm{mp}-22898)$ & 1.6 & 12.3 \\
\hline $\mathrm{RbF}(\mathrm{mp}-11718)$ & 2.8 & 9.6 \\
\hline $\mathrm{RbF}(\mathrm{mp}-11718)$ & 2.8 & 9.6 \\
\hline $\mathrm{RbCl}(\mathrm{mp}-23295)$ & 2.2 & 12.9 \\
\hline $\mathrm{RbBr}(\mathrm{mp}-22867)$ & 5.3 & 28.8 \\
\hline $\mathrm{RbI}(\mathrm{mp}-22903)$ & 2.5 & 20.3 \\
\hline $\mathrm{AgCl}(\mathrm{mp}-22922)$ & 0.7 & 0.9 \\
\hline $\mathrm{AgBr}(\mathrm{mp}-23231)$ & 0.4 & 1.2 \\
\hline $\mathrm{CsF}(\mathrm{mp}-1784)$ & 2.4 & 8.2 \\
\hline $\mathrm{MgO}(\mathrm{mp}-1265)$ & 57.4 & 35.2 \\
\hline $\mathrm{CaO}(\mathrm{mp}-2605)$ & 24.7 & 22.8 \\
\hline $\mathrm{CaS}(\mathrm{mp}-1672)$ & 38.0 & 57.6 \\
\hline $\mathrm{CaSe}(\mathrm{mp}-1415)$ & 23.1 & 46.7 \\
\hline $\mathrm{CaTe}(\mathrm{mp}-1519)$ & 12.4 & 41.6 \\
\hline $\mathrm{SrO}(\mathrm{mp}-2472)$ & 10.3 & 13.8 \\
\hline $\mathrm{BaO}(\mathrm{mp}-1342)$ & 5.2 & 5.5 \\
\hline & &
\end{tabular}

\begin{tabular}{|c|c|c|}
\hline BaS(mp-1500) & 4.8 & 21.4 \\
\hline $\mathrm{CdO}(\mathrm{mp}-1132)$ & 11.1 & 8.9 \\
\hline $\mathrm{PbS}(\mathrm{mp}-21276)$ & 3.7 & 2.8 \\
\hline$\overline{\mathrm{PbSe}(\mathrm{mp}-2201)}$ & 1.3 & 2.8 \\
\hline $\mathrm{PbTe}(\mathrm{mp}-19717)$ & 1.2 & 4.3 \\
\hline $\mathrm{AlN}(\mathrm{mp}-1700)$ & 267.2 & 177.9 \\
\hline BAs(mp-10044) & 1537.8 & 1389.0 \\
\hline $\mathrm{BeO}(\mathrm{mp}-1778)$ & 527.5 & 194.2 \\
\hline $\mathrm{BeSe}(\mathrm{mp}-1541)$ & 239.6 & 255.2 \\
\hline$\overline{\mathrm{BeTe}}(\mathrm{mp}-252)$ & 200.0 & 351.7 \\
\hline $\mathrm{BN}(\mathrm{mp}-1639)$ & 1192.7 & 319.1 \\
\hline $\mathrm{BP}(\mathrm{mp}-1479)$ & 449.8 & 301.3 \\
\hline $\mathrm{CuCl}(\mathrm{mp}-22914)$ & 1.3 & 7.7 \\
\hline CuI(mp-22895) & 6.1 & 39.8 \\
\hline GaN (mp-830) & 201.4 & 202.4 \\
\hline $\mathrm{SiC}(\mathrm{mp}-8062)$ & 471.5 & 317.4 \\
\hline $\mathrm{ZnO}(\mathrm{mp}-1986)$ & 49.9 & 70.6 \\
\hline AlAs(mp-8881) & 83.0 & 199.0 \\
\hline $\mathrm{CdO}(\mathrm{mp}-1132)$ & 11.1 & 8.9 \\
\hline PbS(mp-21276) & 3.7 & 2.8 \\
\hline PbSe(mp-2201) & 1.3 & 2.8 \\
\hline$\overline{\mathrm{PbTe}(\mathrm{mp}-19717)}$ & 1.2 & 4.3 \\
\hline $\mathrm{AlN}(\mathrm{mp}-1700)$ & 267.2 & 177.9 \\
\hline$\overline{\mathrm{BAs}}(\mathrm{mp}-10044)$ & 1537.8 & 1389.0 \\
\hline $\mathrm{BeO}(\mathrm{mp}-1778)$ & 527.5 & 194.2 \\
\hline$\overline{B e S e}(\mathrm{mp}-1541)$ & 239.6 & 255.2 \\
\hline $\operatorname{BeTe}(\mathrm{mp}-252)$ & 200.0 & 351.7 \\
\hline $\mathrm{BN}(\mathrm{mp}-1639)$ & 1192.7 & 319.1 \\
\hline $\mathrm{BP}(\mathrm{mp}-1479)$ & 449.8 & 301.3 \\
\hline $\mathrm{CuCl}(\mathrm{mp}-22914)$ & 1.3 & 7.7 \\
\hline CuI(mp-22895) & 6.1 & 39.8 \\
\hline GaN(mp-830) & 201.4 & 202.4 \\
\hline$\overline{\mathrm{SiC}(\mathrm{mp}-8062)}$ & 471.5 & 317.4 \\
\hline $\mathrm{ZnO}(\mathrm{mp}-1986)$ & 49.9 & 70.6 \\
\hline $\operatorname{AlAs}(m p-8881)$ & 83.0 & 199.0 \\
\hline $\operatorname{AlN}(\mathrm{mp}-661)$ & 243.4 & 173.2 \\
\hline$\overline{\mathrm{BAs}(\mathrm{mp}-984718)}$ & 1169.7 & 1043.6 \\
\hline $\mathrm{BeO}(\mathrm{mp}-2542)$ & 392.9 & 165.5 \\
\hline $\mathrm{BN}(\mathrm{mp}-2653)$ & 965.8 & 277.1 \\
\hline$\overline{\mathrm{BP}}(\mathrm{mp}-1008559)$ & 355.5 & 240.3 \\
\hline $\mathrm{CdS}(\mathrm{mp}-672)$ & 19.8 & 81.6 \\
\hline CdTe(mp-12779) & 4.8 & 42.5 \\
\hline $\mathrm{CuCl}(\mathrm{mp}-1184046)$ & 1.1 & 7.8 \\
\hline GaAs(mp-8883) & 31.2 & 91.0 \\
\hline GaN(mp-804) & 204.4 & 222.0 \\
\hline $\mathrm{GaP}(\mathrm{mp}-8882)$ & 80.7 & 190.8 \\
\hline $\mathrm{SiC}(\mathrm{mp}-7140)$ & 389.0 & 293.3 \\
\hline$\overline{\mathrm{ZnO}(\mathrm{mp}-2133)}$ & 43.7 & 63.5 \\
\hline ZnS(mp-560588) & 23.0 & 76.9 \\
\hline ZnSe(mp-380) & 16.1 & 57.9 \\
\hline$\overline{\mathrm{AgSbBa}(\mathrm{mp}-984720)}$ & 0.4 & 2.7 \\
\hline CoAsZr(mp-961689) & 36.5 & 42.8 \\
\hline CoSbZr(mp-22377) & 39.8 & 44.9 \\
\hline NiScSb(mp-3432) & 4.7 & 6.8 \\
\hline $\mathrm{BeO}(\mathrm{mp}-1778)$ & 527.5 & 194.2 \\
\hline$\overline{\mathrm{BeSe}(\mathrm{mp}-1541)}$ & 239.6 & $\overline{255.2}$ \\
\hline $\mathrm{BeTe}(\mathrm{mp}-252)$ & 200.0 & 351.7 \\
\hline $\mathrm{BN}(\mathrm{mp}-1639)$ & 1192.7 & 319.1 \\
\hline $\mathrm{BP}$ & 449.8 & 301.3 \\
\hline
\end{tabular}




\begin{tabular}{|c|c|c|}
\hline $\mathrm{CuCl}(\mathrm{mp}-22914)$ & 1.3 & 7.7 \\
\hline $\mathrm{CuI}(\mathrm{mp}-22895)$ & 6.1 & 39.8 \\
\hline $\mathrm{GaN}(\mathrm{mp}-830)$ & 201.4 & 202.4 \\
\hline $\mathrm{SiC}(\mathrm{mp}-8062)$ & 471.5 & 317.4 \\
\hline $\mathrm{ZnO}(\mathrm{mp}-1986)$ & 49.9 & 70.6 \\
\hline $\operatorname{AlAs}(m p-8881)$ & 83.0 & 199.0 \\
\hline $\mathrm{CdO}(\mathrm{mp}-1132)$ & 11.1 & 8.9 \\
\hline $\mathrm{PbS}(\mathrm{mp}-21276)$ & 3.7 & 2.8 \\
\hline NiSnTi(mp-924130) & 7.7 & 12.0 \\
\hline NiSnTi(mp-623646) & 7.7 & 12.0 \\
\hline NiSnTi(mp-22782) & 7.7 & 12.0 \\
\hline NiSnZr(mp-924129) & 28.1 & 39.9 \\
\hline NiSnZr(mp-30806) & 28.1 & 39.9 \\
\hline $\begin{array}{l}\mathrm{PdSrTe}(\mathrm{mp}-961663) \\
\end{array}$ & 0.5 & 3.2 \\
\hline PtGaTa(mp-961677) & 47.1 & 84.2 \\
\hline$\overline{\mathrm{PtGeTi}(\mathrm{mp}-1008680)}$ & 7.8 & 11.7 \\
\hline PtGeTi(mp-961671) & 7.8 & 11.7 \\
\hline $\begin{array}{l}\text { PtNbIn(mp-961708) } \\
\text { (mplo }\end{array}$ & 18.6 & 49.4 \\
\hline RhNbSi(mp-1100400) & $\overline{24.2}$ & 26.5 \\
\hline RhSbHf(mp-10367) & 29.8 & 52.3 \\
\hline RuAsV(mp-1100404) & 11.6 & 11.5 \\
\hline SiSrCd(mp-962076) & 4.4 & 7.7 \\
\hline SnSrBa(mp-962062) & 2.4 & 14.2 \\
\hline GeC(mp-1002164) & 776.3 & 376.9 \\
\hline Lonsdaleite(mp-47) & 2159.6 & 821.4 \\
\hline $\mathrm{BC}_{2} \mathrm{~N}(\mathrm{mp}-30148)$ & 1112.1 & 679.2 \\
\hline SiSn(mp-1009813) & 151.1 & 317.0 \\
\hline BSb(mp-997618) & 379.5 & 494.7 \\
\hline $\mathrm{B}_{2} \mathrm{AsP}(\mathrm{mp}-1008528)$ & 433.5 & 576.6 \\
\hline $\mathrm{C}_{3} \mathrm{~N}_{4}(\mathrm{mp}-571653)$ & 334.4 & 387.9 \\
\hline $\mathrm{BC}_{2} \mathrm{~N}(\mathrm{mp}-629458)$ & 993.8 & 581.5 \\
\hline $\mathrm{BeCN}_{2}(\mathrm{mp}-15703)$ & 416.3 & 207.2 \\
\hline $\mathrm{C}_{3} \mathrm{~N}_{4}(\mathrm{mp}-2852)$ & 300.6 & 124.1 \\
\hline SnC(mp-1009820) & 261.7 & 308.1 \\
\hline B(mp-160) & 62.5 & 44.3 \\
\hline LiBC(mp-9244) & 130.0 & 39.6 \\
\hline
\end{tabular}

The full list of materials identified by the machine learning models fulfilling the condition $\ell>\gamma \kappa$ are:

$\mathrm{Se}(\mathrm{mp}-147), \quad \mathrm{Sm}_{2} \mathrm{O}_{3}(\mathrm{mp}-218), \quad \mathrm{YbSe}(\mathrm{mp}-(286)$, $\mathrm{B}_{2} \mathrm{O}_{3}$ (mp-306), $\quad \mathrm{MgP}_{4}(\mathrm{mp}-384), \quad \mathrm{Sc}_{2} \mathrm{~S}_{3}(\mathrm{mp}-401)$, $\mathrm{CdP}_{2}$ (mp-402), $\mathrm{AlF}_{3}(\mathrm{mp}-468), \mathrm{GeO}_{2}(\mathrm{mp}-470), \mathrm{NiP}_{2}(\mathrm{mp}-$ 486), $\quad \mathrm{Ga}_{2} \mathrm{~S} 3(\mathrm{mp}-539), \quad \mathrm{TeO}_{2}(\mathrm{mp}-557), \quad \mathrm{GaF}_{3}(\mathrm{mp}-$ 588), $\quad \mathrm{B}_{6} \mathrm{As}(\mathrm{mp}-624), \quad \mathrm{SnSe}_{2}(\mathrm{mp}-665), \quad \mathrm{GeSe}(\mathrm{mp}-$
700), $\quad \mathrm{P}_{2} \mathrm{Pt}(\mathrm{mp}-730), \quad \mathrm{Li}_{3} \mathrm{P}(\mathrm{mp}-736), \quad \mathrm{Li}_{3} \mathrm{As}(\mathrm{mp}-757)$, $\mathrm{Ga}_{2} \mathrm{O} 3(\mathrm{mp}-886), \quad \mathrm{As}_{2} \mathrm{Se}_{3}$ (mp-909), $\quad \mathrm{CuP}_{2}$ (mp-927), GeTe(mp-938), $\quad$ NbZn $_{3}(\mathrm{mp}-953), \quad \mathrm{K}_{2} \mathrm{O}(\mathrm{mp}-971)$, $\mathrm{SrF}_{2}$ (mp-981), $\quad \mathrm{BaF}_{2}$ (mp-1029), $\quad \mathrm{SrS}(\mathrm{mp}-1087)$, $\mathrm{Al}_{2} \mathrm{O}_{3}$ (mp-1143), $\quad \mathrm{ThS}_{2}$ (mp-1146), $\mathrm{YbO}(\mathrm{mp}-1216)$, $\mathrm{MgS}(\mathrm{mp}-1315), \quad \mathrm{Ga}_{2} \mathrm{Se}_{3}(\mathrm{mp}-1340), \quad \mathrm{ZnP}_{2}(\mathrm{mp}-1392)$, $\mathrm{Rb}_{2} \mathrm{O}(\mathrm{mp}-1394), \quad \mathrm{Sn}_{2} \mathrm{~S}_{3}$ (mp-1509), $\quad \mathrm{As}_{2} \mathrm{O}_{3}(\mathrm{mp}-1581)$, $\mathrm{Al}_{4} \mathrm{C}_{3}$ (mp-1591), $\quad \mathrm{Tm}_{2} \mathrm{O} 3(\mathrm{mp}-1767), \quad \mathrm{YbTe}(\mathrm{mp}-1779)$, $\mathrm{SiF}_{4}(\mathrm{mp}-1818), \quad \mathrm{YbS}(\mathrm{mp}-1820), \quad \mathrm{SiAs}(\mathrm{mp}-1863)$, $\mathrm{ZnF}_{2}$ (mp-1873), $\quad \mathrm{SrTe}(\mathrm{mp}-1958), \quad \mathrm{Mg}_{3} \mathrm{As}_{2}$ (mp-1990), $\mathrm{Li}_{3} \mathrm{Sb}(\mathrm{mp}-2074), \quad \mathrm{Rb}_{2} \mathrm{P}_{3}(\mathrm{mp}-2079), \quad \mathrm{Si}_{5} \mathrm{Ir}_{3}(\mathrm{mp}-2084)$, $\mathrm{KGe}(\mathrm{mp}-2146), \quad \mathrm{Er}_{2} \mathrm{~S} 3(\mathrm{mp}-2234), \quad \mathrm{GeS}(\mathrm{mp}-2242)$, $\mathrm{Tm}_{2} \mathrm{~S}_{3}$ (mp-2309), $\quad \mathrm{Dy}_{2} \mathrm{O}_{3}(\mathrm{mp}-2345), \quad \mathrm{Ga}_{2} \mathrm{Te}_{5}(\mathrm{mp}-$ 2371), $\quad \mathrm{NaSi}(\mathrm{mp}-2402), \quad \mathrm{P}_{4} \mathrm{Se}_{5}(\mathrm{mp}-2447), \quad \mathrm{Al}_{2} \mathrm{~S}_{3}$ (mp2654), $\mathrm{CaF}_{2}$ (mp-2741), SrSe(mp-2758), SiP(mp-2798), $\mathrm{Lu}_{2} \mathrm{~S}_{3}$ (mp-2826), $\quad \mathrm{InF}_{3}(\mathrm{mp}-6949), \quad \mathrm{Be}_{3} \mathrm{~N}_{2}$ (mp-6977), $\mathrm{Rb}_{2} \mathrm{Se}_{3}(\mathrm{mp}-7447), \quad \mathrm{GeF}_{2}(\mathrm{mp}-7595), \mathrm{K}_{2} \mathrm{Se}_{3}(\mathrm{mp}-7670)$, $\mathrm{ThSe}_{3}(\mathrm{mp}-7951), \quad \mathrm{PF}_{5}$ (mp-8511), $\quad \mathrm{AsF}_{5}(\mathrm{mp}-8723)$, $\mathrm{Al}_{2} \mathrm{Te}_{5}$ (mp-9254), GeAs(mp-9548), ZnS(mp-9946), $\mathrm{MgSe}(\mathrm{mp}-10760), \quad \mathrm{RbSn}(\mathrm{mp}-11054), \quad \mathrm{Sr}_{3} \mathrm{As}_{4}(\mathrm{mp}-$ 15339), $\quad \mathrm{Ge}_{3} \mathrm{Os}_{2}(\mathrm{mp}-16610), \quad \mathrm{GeAs}_{2}(\mathrm{mp}-17524)$, InS(mp-19795), InSe(mp-20485), $\quad \mathrm{Si}_{3} \mathrm{Ru}_{2}(\mathrm{mp}-22192)$, $\mathrm{In}_{2} \mathrm{O}_{3}(\mathrm{mp}-22323), \mathrm{CsCl}(\mathrm{mp}-22865), \mathrm{YbBr}_{2}(\mathrm{mp}-22882)$, $\mathrm{XeF}_{2}$ (mp-22885), $\mathrm{CaBr}_{2}$ (mp-22888), $\mathrm{ZnCl}_{2}$ (mp-22889), $\mathrm{CaCl}_{2}$ (mp-22904), $\mathrm{CsBr}(\mathrm{mp}-22906), \mathrm{PtBr}_{3}$ (mp-23165), $\mathrm{IO}_{2}$ (mp-23170), $\quad \mathrm{SrI}_{2}$ (mp-23181), $\quad \mathrm{BaCl}_{2}$ (mp-23199), $\mathrm{InI}(\mathrm{mp}-23202), \quad \mathrm{MgI}_{2}$ (mp-23205), $\quad \mathrm{SrCl}_{2}$ (mp-23209), $\mathrm{MgCl}_{2}$ (mp-23210), $\mathrm{AsI}_{3}$ (mp-23218), $\mathrm{YbCl}_{2}$ (mp-23220), $\mathrm{BaI}_{2}$ (mp-23260), $\mathrm{BeCl}_{2}$ (mp-23267), $\quad \mathrm{BeP}_{2}$ (mp-27148), $\mathrm{ZnI}_{2}$ (mp-27161), $\mathrm{BaBr}_{2}$ (mp-27456), $\mathrm{Te}_{3} \mathrm{Cl}_{2}$ (mp-27628), $\mathrm{ThI}_{4}$ (mp-27697), $\quad \mathrm{PdI}_{2}$ (mp-27747), $\quad \mathrm{GeI}_{2}$ (mp-27922), $\mathrm{CdI}_{2}$ (mp-28248), $\quad \mathrm{P}_{2} \mathrm{Pd}(\mathrm{mp}-28266), \quad \mathrm{PtI}_{2}$ (mp-28319), $\mathrm{Pt}_{3} \mathrm{I}_{8}$ (mp-28320), $\mathrm{GaBr}_{2}$ (mp-28384), $\mathrm{Br}_{2} \mathrm{O}$ (mp-28460), $\mathrm{In}_{7} \mathrm{Cl}_{9}$ (mp-28730), $\mathrm{Ca}_{5} \mathrm{P}_{8}$ (mp-28879), $\mathrm{Cl}_{2} \mathrm{O}$ (mp-29537), $\mathrm{NaGe}(\mathrm{mp}-29657), \mathrm{Tl}_{2} \mathrm{Te}_{3}$ (mp-29711), $\mathrm{LaAs}_{2}$ (mp-29815), $\mathrm{CaI}_{2}(\mathrm{mp}-30031), \quad \mathrm{MgBr}_{2}(\mathrm{mp}-30034), \quad \mathrm{BeI}_{2}(\mathrm{mp}-30140)$, $\mathrm{GaCl}_{3}$ (mp-30952), $\mathrm{Si}_{9} \mathrm{Te}_{8}$ (mp-31135), $\mathrm{NbI}_{5}$ (mp-31487), $\mathrm{Tm}_{2} \mathrm{Se}_{3}(\mathrm{mp}-32850), \quad \mathrm{AuBr}(\mathrm{mp}-505366), \quad \mathrm{Cs}_{2} \mathrm{~S}(\mathrm{mp}-$ 540703), KSn(mp-542374), $\quad \mathrm{ThCl}_{4}(\mathrm{mp}-567431)$, $\mathrm{SrBr}_{2}(\mathrm{mp}-567744), \mathrm{HfI}_{4}(\mathrm{mp}-569059), \mathrm{SiB}_{3}(\mathrm{mp}-569128)$, $\mathrm{TeI}(\mathrm{mp}-569766), \mathrm{ThBr}_{4}(\mathrm{mp}-570229), \mathrm{YbI}_{2}(\mathrm{mp}-570418)$, $\mathrm{SeBr}(\mathrm{mp}-570589), \quad \mathrm{Ga}_{3} \mathrm{Os}(\mathrm{mp}-570844), \quad \mathrm{ZrI}_{4}(\mathrm{mp}-$ 571235), $\quad \mathrm{Ga}_{2} \mathrm{I}_{3}(\mathrm{mp}-636675), \quad \mathrm{In}_{7} \mathrm{Te}_{1} 0$ (mp-669311), $\mathrm{Lu}_{2} \mathrm{Se}_{3}(\mathrm{mp}-673650), \quad \mathrm{Sc}_{2} \mathrm{Se}_{3}(\mathrm{mp}-684690), \quad \mathrm{CsSi}(\mathrm{mp}-$ 866482), SiGe(mp-978534).
[1] R. A. Guyer and J. A. Krumhansl, Phys. Rev. 148, 766 (1966).

[2] K. Esfarjani and H. Stokes, Phys. Rev. B
77, 144112 (2008), ISSN 1098-0121, URL http://link.aps.org/doi/10.1103/PhysRevB.77.144112 [3] L. Chaput, Phys. Rev. Lett. 110, 265506 (2013), 
1303.4062.

[4] P. Torres, Thermal Transport in Semiconductors: First Principles and Phonon Hydrodynamics (Springer Nature, Switzerland, 2018).

[5] J. A. Johnson, A. A. Maznev, J. Cuffe, J. K. Eliason, A. J. Minnich, T. Kehoe, C. M. S. Torres, G. Chen, and K. A. Nelson, Phys. Rev. Lett. 110, 025901 (2013), ISSN 00319007, 1204.4735, URL http://link.aps.org/doi/10.1103/PhysRevLett.110.025962:

[6] M. E. Siemens, Q. Li, R. Yang, K. K. A. Nelson, E. H. Anderson, M. M. Murnane, and H. C. Kapteyn, Nat. Mater. 9, 26 (2010), ISSN 1476-1122, URL http://www . nature.com/nmat/journal/v9/n1/abs/nmat

[7] A. J. Minnich, J. A. Johnson, A. J. Schmidt, K. Esfarjani, M. S. Dresselhaus, K. A. Nelson, and G. Chen, Phys. Rev. Lett. 107 (2011), ISSN 00319007, URL \{\%\}3CGoto.

[8] K. T. Regner, D. P. Sellan, Z. Su, C. H. Amon, A. J. H. McGaughey, and J. Malen, Nature Comm. 4, 1640 (2013), ISSN 2041-1723, URL http://www.ncbi.nlm.nih.gov/pubmed/23535661

[9] R. B. Wilson and D. G. Cahill, Nature Comm. 5, 5075 (2014), ISSN 2041-1723, URL http://www.ncbi.nlm.nih.gov/pubmed/25270394

[10] Y. Hu, L. Zeng, A. J. Minnich, M. S. Dresselhaus, and G. Chen, Nat. Nanotechnol. 10, 701 (2015), ISSN 1748-3387, URL http://www . scopus. com/inward/record.url?eid=2-s2.0-

[11] K. M. Hoogeboom-Pot, J. N. Hernandez-Charpak, X. Gu, T. D. Frazer, E. H. Anderson, W. Chao, R. W. Falcone, R. Yang, M. M. Murnane, H. C. Kapteyn, et al., Proc. Natl. Acad. Sci. 112, 201503449 (2015), ISSN 0027-8424, URL http://www .pnas.org/lookup/doi/10.1073/pnas.1503449112

[12] B. Vermeersch, A. M. S. Mohammed, G. Pernot, Y. R. Koh, and A. Shakouri, Phys. Rev. B 90, 1 (2014), ISSN $1550235 \mathrm{X}$.

[13] B. Vermeersch and A. Shakouri, arXiv:1412.6555 pp. 1-21 (2014), 1412.6555, URL http://arxiv.org/abs/1412.6555.

[14] A. Cepellotti, G. Fugallo, L. Paulatto, M. Lazzeri, F. Mauri, and N. Marzari, Nat. Commun. 6, 6400 (2015).

[15] S. Lee, D. Broido, K. Esfarjani, and G. Chen, Nat. Commun. 6, 6290 (2015).

[16] P. Torres, A. Ziabari, A. Torelló, J. Bafaluy, J. Camacho, X. Cartoixà, A. Shakouri, and F. X. Alvarez, Phys. Rev. Materials 2, 076001 (2018).

[17] R. A. Guyer and J. A. Krumhansl, Phys. Rev. 148, 778 (1966).

[18] A. Ziabari, P. Torres, B. Vermeersch, X. Cartoixà, A. Torelló, Y. Xuan, J.-H. Bahk, Y. Koh, Y. P., F. Alvarez, et al., Nat. Commun. 9, 255 (2018).

[19] Y. R, XenonPy (2020), URL https://github.com/yohida-lab/XenonPy

[20] A. Jain, Y. J. Yu, and A. J. H. McGaughey, Phys. Rev. B 87, 1 (2013), ISSN 10980121.

[21] S. P. Ong, S. Cholia, A. Jain, M. Brafman, D. Gunter, G. Ceder, and K. A. Persson, Computational Materials Science 97, 209 (2015), ISSN 0927-0256, URL
http://www.sciencedirect.com/science/article/pii/S092702561

[22] A. Seko, A. Togo, H. Hayashi, K. Tsuda, L. Chaput, and I. Tanaka, Phys. Rev. Lett. 115, 205901 (2015), URL https://link.aps.org/doi/10.1103/PhysRevLett.115.205901

[23] J. Carrete, W. Li, N. Mingo, S. Wang, and S. Curtarolo, Phys. Rev. X 4, 011019 (2014), URL https://link.aps.org/doi/10.1103/PhysRevX.4.011019.

[24] A. Togo and I. Tanaka, Scr. Mater. 108, 1 (2015).

A. Togo, L. Chaput, and I. Tanaka, Phys. Rev. B 91, 094306 (2015).

[26] P. Torres, F. Alvarez, and X. Cartoixa, Kinetic Collective Model: BTE-based hydrodynamic model for thermal 568.htmiansport (2020), URL https://physta.github.io/

[27] R. Juneja, G. Yumnam, S. Satsangi, and A. K. Singh, Chem. Mater. 31, 5145 (2019).

[28] S. Ju, R. Yoshida, C. Liu, K. Hongo, T. Tadano, and J. Shiomi (2020), URL https://arxiv.org/abs/1909.11234

[29] L. Lindsay, Phys. Rev. B 94, 174304 (2016), URL https://link.aps.org/doi/10.1103/PhysRevB.94.174304

[30] A. Ward and D. A. Broido, Phys. Rev. B 81, 085205 (2010), ISSN 1098-0121, arXiv:1107.5288v1, URL http://link.aps.org/doi/10.1103/PhysRevB.81.085205

[31] A. Ward, D. A. Broido, D. A. Stewart, and G. Deinzer, Phys. Rev. B 80, 125203 (2009).

[32] D. A. Broido, L. Lindsay, and T. L. Reinecke, Phys. Rev. 84930B58812\{\&48par(tzelri)D=tZOtx3y1\{\%\}5Cnhttp: //www . nature . com/do if

[33] M. Muñoz Rojo, Y. Zhang, C. V. Manzano, R. Alvaro, J. Gooth, M. Salmeron, and M. Martin-Gonzalez, Sci. Rep. 6, 19014 (2016), ISSN 2045-2322, URL http://www .nature.com/articles/srep19014

[34] M. Muñoz Rojo, B. Abad, C. V. Manzano, P. Torres, X. Cartoixà, F. X. Alvarez, and M. S. Martín-González, Nanoscale 9, 6741 (2017), ISSN 2040-3364, URL http://pubs.rsc.org/en/Content/ArticleLanding/2017/NR/C7NRO

[35] T. Koga, X. Sun, S. Cronin, and M. Dresselhausb, Applied Physics Letters 73 (1998).

[36] V. Maurya, U. Paliwal, G. Sharma, and K. B. Joshi, RSC Adv. 9, 13515 (2019), URL http://dx.doi.org/10.1039/C9RA01573F

[37] H. Hoshino and M. Shimoji, Journal of Physics and Chemistry of Solids 31, 1553 (1970), ISSN 0022-3697, URL https://www.sciencedirect.com/science/article/pii/002236977

[38] Y. Xiao and L.-D. Zhao, npj Quantum Materials 3, 2397 (2018), ISSN 1.

[39] P. Geurts, D. Ernst, and L. Wehenkel, Machine Learning. 63, 3 (2006).

[40] L. Breiman, Machine Learning. 54, 5 (2001).

[41] J. H. Friedman, Computational Statistics and Data Analysis 38, 367 (2002).

[42] Y. J. Han and P. G. Klemens, Phys. Rev. B 48, 6033 (1993), ISSN 01631829.

[43] Y.-J. Han, Phys. Rev. B 54, 8977 (1996), ISSN 01631829.

[44] C. Herring, Phys. Rev. 95, 954 (1954), ISSN 0031899X, URL http://link.aps.org/doi/10.1103/PhysRev.95.954. 
[45] S. I. Tamura, Phys. Rev. B 27, 858 (1983), ISSN 01631829.

[46] P. G. Klemens, Proc. Phys. Soc. A 68, 1113 (1955), ISSN 0370-1298.

[47] P. Klemens, Phys. Rev. 119, 507 (1960), ISSN 0031-899X, URL http://link.aps.org/doi/10.1103/PhysRev.119.507.

[48] A. V. Inyushkin, A. N. Taldenkov, A. Y. Yakubovsky, A. V. Markov, L. MorenoGarsia, and B. N. Sharonov, Semicond. Sci. Tech. 18, 685 (2003), ISSN 02681242, URL http://stacks.iop.org/0268-1242/18/i=7/a=315

[49] A. V. Inyushkin, A. N. Taldenkov, A. M. Gibin, A. V. Gusev, and H.-J. Pohl, Phys. Status Solidi (C) 1, 2995 (2004), ISSN 16101634, URL http://doi.wiley.com/10.1002/pssc. 200405341

[50] P. Torres, J. Íñiguez, and R. Rurali, Phys. Rev. Lett. 123, 185901 (2019), URL https://link.aps.org/doi/10.1103/PhysRevLett.123.

[51] S. P. Ong, W. D. Richards, A. Jain, G. Hautier, M. Kocher, S. Cholia, D. Gunter, V. L. Chevrier, K. A. Persson, and G. Ceder, Computational Materials Science 68, 314 (2013), ISSN 0927-0256, URL http://www.sciencedirect.com/science/article/pii/SO

$\begin{array}{cccr}\text { [52] A. Phonon } & \text { Tatabase } & \text { at } \\ \text { Kyoto } & \text { university } & \text { (2020), }\end{array}$ Kyoto university (2020),
http://phonondb.mtl.kyoto-u.ac.jp/index.html

[53] J. Zhou, B. Liao, B. Qiu, S. Huberman, K. Esfarjani, M. S. Dresselhaus, and G. Chen, Proceedings of the National Academy of Sciences 112, 14777 (2015), ISSN 0027-8424, https://www.pnas.org/content/112/48/14777.full.pdf, URL https : //www . pnas . org/content/112/48/14777

[54] I. Kuleev, I. Kuleev, A. Taldenkov, A. Inyushkin, V. Ozhogin, K. Itoh, and E. Haller, Journal of Experimental and Theoretical Physics 96, 1078 (2003).

[55] A. Levchenko and J. Schmalian, Annals of Physics 419, 168218 (2020), ISSN 0003-4916, URL https://www.sciencedirect.com/science/article/pii/S0003491620301524.
[56] J. Gooth, F. Menges, N. Kumar, V. SüB, C. Shekhar, Y. Sun, U. Drechsler, R. Zierold, C. Felser, and B. Gotsmann, Nat. Commun. 9, 4093 (2018).

[57] I. Kuleyev, I. Kuleyev, S. Bakharev, and V. Ustinov, J. Exp. Theor. Phys. 123, 489 (2016), ISSN 0003-4916.

[58] X. Huang and A. Lucas, Phys. Rev. B 103, 155128 (2021), URL https://link.aps.org/doi/10.1103/PhysRevB.103.155128

[59] M. M. Glazov, Phys. Rev. B 100, 045426 (2019), URL https://link.aps.org/doi/10.1103/PhysRevB.100.045426

[60] E. Jiang, X. Zhu, T. Ouyang, C. Tang, J. Li, C. He, C. Zhang, and J. Zhong, Journal of Applied Physics 126, 185106 (2019), https://doi.org/10.1063/1.5121517, URL https://doi.org/10.1063/1.5121517.

[61] Z. Azarmi, M. Naseri, and S. Parsamehr, Chemical Physics Letters 728, 136 (2019), ISSN 0009-2614, URL http://www.sciencedirect.com/science/article/pii/S000926141

[62] S. Kansara, Y. Sonvane, P. N. Gajjar, and S. K. Gupta, RSC Adv. 10, 26804 (2020), URL http://dx.doi.org/10.1039/D0RA03599H

[63] T. Zhao, Y. Sun, Z. Shuai, and D. Wang, Chemistry of Materials 29, $6261 \quad$ (2017), https://doi.org/10.1021/acs.chemmater.7b01343, URL

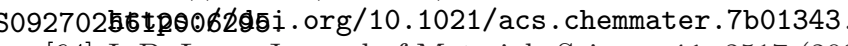

[64] I. B. Lucy, Journal of Materials Science 41, 2517 (2006).

[65] M. L. Snedaker, Alternative Approaches to Group IV Thermoelectric Materials ( $\mathrm{PhD}$ dissertation, University of California, Santa Barbara, 2015).

[66] S. Narushima, H. Hosono, J. Jisun, T. Yoko, and K. Shimakawa, Journal of Non-Crystalline Solids 274, 313 (2000), ISSN 0022-3093, physics of Non-Crystalline Solids 9, URL http://www.sciencedirect.com/science/article/pii/S002230930

[67] Y. Shiraishi, S. Hata, Y. Okawauchi, K. Oshima, H. Anno, and N. Toshima, Chemistry Letters 46, 933 (2017), https://doi.org/10.1246/cl.170218, URL https://doi.org/10.1246/cl.170218 